\title{
Improving documentation of treatment escalation decisions in acute care
}

Mark Dahill, Louise Powter, Lynn Garland, Mark Mallett, Jerry Nolan

\begin{abstract}
The aim of this project was to improve the documentation of treatment escalation decisions at a district general hospital in southwest England. A pilot 'Ceiling of Treatment' proforma was trialled on the care of the elderly wards at the Royal United Hospital (RUH), Bath. Successive PDSA cycles enabled revision of the proforma for use across the Trust. Data were collected on the proportion of patients with a documented treatment escalation decision. Formative feedback was collected via questionnaire from trainees and discussion with special interest groups of consultants within the hospital. This approach involved collaboration between acute medicine, intensive care, elderly care, the resuscitation department, palliative care and the legal department. Documentation of ceiling of treatment decisions rose from $30 \%$ to $90 \%$ during the study. A survey of medical trainees showed $67 \%(n=36)$ had seen the ceiling of treatment form, of which, $100 \%$ found it useful on on-call shifts. Initiating a proforma to record treatment escalation decisions and combining this with the existing 'Do not attempt cardiopulmonary resuscitation' (DNAR) paperwork, increased decision making and documentation. This intervention ensures patients receive the appropriate level of care, as indicated by their consultant, and reduces anxiety for junior doctors during on-call shifts.
\end{abstract}

\section{Problem}

Patients are often subjected to distressing investigations and treatments, inappropriate to their presentation, by on-call doctors who are forced to make treatment escalation decisions without detailed knowledge of the patient's circumstances. This problem arises because of difficulties in documenting treatment limits in the medical notes, and inherent lack of continuity as a result of on-call cover. At the RUH, there was no standardised method for recording these important decisions in the medical notes.

\section{Background}

Treatment escalation describes an increasing the complexity of care for patients up to a designated 'ceiling of treatment'. An experienced clinician, with knowledge of both the patient and problem, often after discussion with the patient and family, can designate an appropriate ceiling to treatment. A quality improvement project was undertaken to improve decision making and documentation of escalation of treatment, in line with the recent NCEPOD report (1). The work took place at the RUH, a medium sized district general hospital in South West England. The RUH previously used a DNAR form - an intervention at the very end of a spectrum of treatment options. Often no ceiling of treatment decision was documented in patients' medical notes, leading to inappropriate treatment/investigation and difficult decisions for outof-hours doctors.

The first-hand experience of the authors - having to make difficult out-of-hours treatment decisions without clear guidance from the medical team caring for the patient - was a key motivator for this project. One study documented $96 \%$ of patients regarded treatment escalation planning as a positive component of their care (2), and current guidelines advocate the use of a ceiling of treatment approach in acute care (3).

\section{Baseline Measurement}

Alternate sets of notes on the care of the elderly wards were analysed for presence or absence of a documented treatment escalation decision. At baseline, these were mostly weekend plans or specific overnight instructions based on outstanding investigations. The documentation rate for the 57 patients included was $30 \%$.

See supplementary file: ds2024.pdf - "figure_one"

\section{Design}

The intervention was an easy to use form, clearly showing a ceiling of treatment for each patient (see figure one). The appearance and content of the form was created after discussion with trainees, consultants, nurses and allied healthcare professionals.

The form was made widely available on the care of the elderly wards and data were again collected for presence of a documented ceiling of treatment decision in the medical notes of each patient. Questionnaire data, collected via email to all medical trainees in the Trust, provided formative feedback for evolution of the form.

The results of improved documentation rates, and a refined form incorporating DNAR decisions, were presented to the care of the elderly consultant team and a special interest consultant panel. After further changes at these levels, the form was discussed by the Documents and Legal Review Panel and subsequently instituted as trust protocol for all patients.

\section{Strategy}

PDSA Cycle 1

The initial documentation rate for a treatment escalation decision 
was $30 \%$ for patients on the care of the elderly wards. The plan was to derive an easy to use document for describing treatment escalation decisions. The form was made available on the intervention wards with verbal instructions to all medical staff at the weekly meeting "to use the form if they considered it helpful". No training was given about its use. After 6 weeks of intervention, documentation rates on the intervention wards improved to $57 \%$ $(\mathrm{n}=113$ patients).

PDSA Cycle 2

A 'Survey Monkey' questionnaire was sent to all trust medical trainees via email. There were 70 respondents to this questionnaire. $67 \%$ of trainees (46) had seen the ceiling of treatment form during their on-call shifts. $100 \%$ of respondents who had seen the form considered it 'useful'. Feedback was predominantly positive, mainly describing ease of decision making and time saved when reviewing patients.

The main negative feedback concerned replication of information on existing proformas (for example, post-take ward round proforma) and potential overlap with the established DNAR paperwork. The actions during this cycle of change were to combine the DNAR and ceiling of treatment forms and include detailed instructions for use on the reverse.

\section{Results}

The refined and merged form was modified and reviewed at trust legal and consultant level. This was then published as trust protocol for recording resuscitation and treatment escalation decisions (see figure two).

Data were collected from the same intervention wards after change of the trust protocol, for the presence or absence of a treatment escalation decision in the medical notes. The observed documentation rate increased to $90 \%$ ( $n=113$ patients).

See supplementary file: ds2023.pdf - "figure_two"

\section{Lessons and Limitations}

A key lesson from the project was the inclusion of questionnaire data. The data provided invaluable formative feedback for improvement of the form and also gave specific examples and accounts to illuminate its use. When presenting to senior clinicians and decision makers, accounts from the trainee perspectives provided great insight. Presenting data on improved documentation was key; however individuals accounts of the improvements the form had made to patient care were both emotive and enlightening.

The evolution of the ceiling of treatment form is not specific to the RUH; many trusts in the UK are now adopting similar approaches (4). The approach and findings of this project are widely applicable to trusts across the UK.

An important limitation to this work is a lack of evidence about the impact on patients and carers. Questionnaire testimony described reduced patient anxiety and eased decision making for doctors, based solely on the opinion of doctors. This study did not collect feedback from the patients' perspective and whether the advent of the ceiling of treatment form improved their experience. This was considered outside the remit of this study but is an important consideration when striving to include patients and carers in the decision-making process.

\section{Conclusion}

Using a designated ceiling of treatment form, and ultimately combining it with a DNAR form, improved the documentation rates of treatment escalation decisions. Rates increased from $30 \%$ to $90 \%$, which implies more patients will receive an appropriate level of care in a timely manner. The change of policy has had a lasting effect on patient care at the RUH as out of hours doctors now have consultant endorsed treatment plans for the majority of patients, thus improving patient safety and care.

\section{References}

1. National Confidential Enquiry into Patient Outcome and Death (2012) Time to Intervene

2. Obolensky L, Clark T, Matthew G, Mercer M A patient and relative centred evaluation of treatment escalation plans: a replacement for the do-not-resuscitate process. Journal Medical Ethics 2010; 36(9):518-20

3. The College of Emergency Medicine - clinical effectiveness committee (2012) End of life care for adults in the Emergency department - best practice guidance.

4. Mercer M Treatment escalation plans BMJ 2009;338:b2020

\section{Declaration of interests}

Nothing to declare 